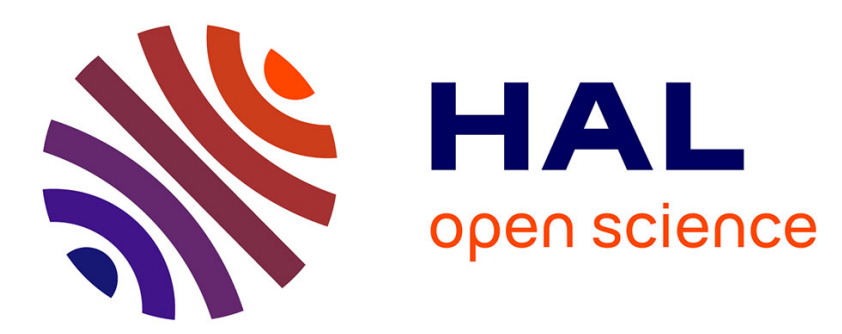

\title{
Robust and GPU-accelerated IPO to improve high frequency EM scattering from large scale radar scene simulation
}

\author{
A. Pajot, N. Douchin, H.J. Mametsa
}

\section{- To cite this version:}

A. Pajot, N. Douchin, H.J. Mametsa. Robust and GPU-accelerated IPO to improve high frequency EM scattering from large scale radar scene simulation. Radar 2014, Oct 2014, LILLE, France. hal01094352

\section{HAL Id: hal-01094352 \\ https://hal.science/hal-01094352}

Submitted on 12 Dec 2014

HAL is a multi-disciplinary open access archive for the deposit and dissemination of scientific research documents, whether they are published or not. The documents may come from teaching and research institutions in France or abroad, or from public or private research centers.
L'archive ouverte pluridisciplinaire HAL, est destinée au dépôt et à la diffusion de documents scientifiques de niveau recherche, publiés ou non, émanant des établissements d'enseignement et de recherche français ou étrangers, des laboratoires publics ou privés. 


\section{Robust and GPU-accelerated IPO to improve high frequency EM scattering from large scale radar scene simulation}

\author{
Anthony Pajot \\ Oktal-SE \\ Toulouse, France \\ Email: Anthony.Pajot@oktal-se.fr
}

\author{
Nicolas Douchin \\ Oktal-SE \\ Toulouse, France \\ Email: Nicolas.Douchin@oktal-se.fr
}

\author{
Henri-José Mametsa \\ ONERA \\ Toulouse, France \\ Email: Henri-Jose.Mametsa@onera.fr
}

\begin{abstract}
Tools for electromagnetic simulation make EM computations down to millimeter wavelength possible from large complex targets and their environments. In this paper, we present how we add localized support for $\mathrm{N}-\mathrm{OP}$, most useful for cavities and other complex objects subject to multiple reflections, to our shooting-and-bouncing-ray SE-RAY-EM software. This extension is made with the Iterative Physical Optics (IPO) method, which we improve to make it suitable for use in intensive EM simulation of large scale scenes with localized N-OP bounces accuracy. As we present, this requires a generalization in materials to handle arbitrary dielectric coatings, numerical robustness, computational efficiency, which we improve by a factor 1600 on high-end GPUs and 300 on laptop GPUs, and a more precise hybridization method than existing work.
\end{abstract}

\section{INTRODUCTION}

The SE-RAY-EM software includes several tools for the generation and the management of physically textured databases, the edition of scenarios relating to a given sensor, the post-processing and visualisation of the output data and an EM core. This computer code capitalises the results of research activities in high frequency scattering methods. A unique tool is currently available for studies dealing with radar analysis, antennas radiation, EM inter-system compatibility and propagation applications through the electromagnetic calculations of the interactions between EM wave and the observed scene.

This numerical simulation tool has the ambition to calculate scattered EM fields at high frequencies (i.e. the size of objects is supposed large compared to the wavelength), in a virtual 3D, geometrically and physically complex environment including natural and man made objects. To achieve this goal, SE-RAYEM couples an optimized SBR algorithm with EM models of propagation, reflection and diffraction which allow unified calculation for the near or far EM scattered fields from the scene. These models are the formulations of Geometrical Optics (GO), Physical Optics (PO), Uniform Theory of Diffraction (UTD) and Equivalent Current Method (ECM). Thanks to the optimized SBR technique, the computation time is only slightly dependent on the complexity of the treated virtual scene.

However, SE-RAY-EM does not manage higher-order effects such as creeping waves or multiple diffusion bounces on a same path: only specular reflections can be chained, ended by a diffusion toward the reception point. As SE-RAY-EM is an asymptotic high-frequency model, it does not aim at having the same accuracy as exact methods. However, some effects reachable by asymptotic models such as PO are still ignored, one of them being multiple PO diffusions on a same path. The contributions of these N-PO paths can in general be safely ignored in large scenes, but there are some common and important cases where these contributions have to be taken into account in order to get accurate results, such as cavities.

We make the observation that cavities and other similar objects are localized in a large scene and small compared to the scene, and therefore it is enough to compute N-PO bounces only on very small portions of the scene. This is why we propose to hybridize SE-RAY-EM with an N-PO-bounce method that will be used for small portions of the scene. We have chosen the Iterative Physical-Optics (IPO) method because its design and goals satisfy our objectives. However, as we show in Section II, it can not be directly used but must be improved in several ways, which we present in the same section. We perform several tests and applications, presented in Section III, in order to validate the accuracy and efficiency of the method, and its suitability for bringing N-PO effects into SE-RAY-EM.

\section{ImPRoved ItERATIVE PhysicAl Optics}

In essence, IPO is the iterative refinement of the first-order PO surface currents to incorporate higher-order multi-bounce and multiple diffraction effects. Practically, it is the iterative solution of a simplified magnetic-field integral equation (MFIE), where we ignore tangential components. This makes the crucial difference between methods of moments (MoM) and IPO: in IPO, the current computation is entirely local, ignoring effects such as edge currents, but it allows for much coarser discretizations $(\lambda / 2$ instead of $\lambda / 10)$. The original IPO method [1] is based on a simplified MFIE stated for the equivalent currents on the surface of a perfect electric conducting (PEC) cavity, and uses the Kirchhoff approximation on the cavity aperture to capture the incident wave. As summarized in [2], IPO has been extended in several ways to extend its scope: partially lossy walls can be easily handled [3], larger cavities [4], [5] or more general objects [6] can be handled, and an improved solver is presented [5]. In this paper, we push the generalization a step further. 
- Currently, only materials with highly-absorbing dielectric coatings can be handled. We show in Subsection II-B how to handle materials with arbitrary dielectric coatings on metallic surfaces.

- Even the improved solver [5] can require a large number of iterations, leading to long computation times, or even diverge. Subsection II-C presents a solver that is robust and typically requires a small number of iterations for IPO resolution.

- $\quad$ Propagation operations are the most time-consuming part of the computation, and are subject to numerical inaccuracy for close points at corners, problem which is strengthened with the dielectric formulation we present. Our GPU-based strategy for efficient propagation computations is presented in Subsection II-D, with a controlled accuracy of the integrals computation.

- The Kirchhoff approximation can not reproduce accurately complex incident waves created by multiple reflections or shadowing before reaching the object. These complex incident waves are common when coupling shooting-and-bouncing (SBR) methods with IPO. We present in Subsection II-E a new SBRIPO hybridation method that does not perform any approximation on the incident wave.

\section{A. IPO formulation}

Standard IPO for the PEC case aims at finding the electric current distribution $\mathrm{J}$ on a surface $S$ that satisfies

$$
\begin{aligned}
\bar{J}(\bar{r}) & =2 \hat{n} \times \bar{H}_{i}^{i}+2 \hat{n} \times \bar{H}_{i}(\bar{r}) \\
\bar{H}_{i}(\bar{r}) & =f_{S} \bar{J}\left(\bar{r}^{\prime}\right) \times \nabla G\left(\bar{r}-\bar{r}^{\prime}\right) d S^{\prime}
\end{aligned}
$$

for each point $\bar{r}$ with normal $\hat{n}$ of $S . \bar{H}_{i}^{i}$ is the initial magnetic field induced by the incident wave and incident to the surface, and $\nabla G(\bar{R})$ is the gradient of the free space Green's function, given by

$$
\nabla G(\bar{R})=\hat{R}\left(j k+\frac{1}{R}\right) \frac{e^{-j k R}}{4 \pi R}
$$

where $k=2 \pi / \lambda$ is the wavenumber of the incident wave.

IPO is solved by first discretising the surface in tiny patches, with in average 4 to 9 patches per $\lambda^{2}$, and compute the value of the current on each patch.

The final field at a reception point is then the sum of the field radiated by $\bar{J}$ toward this point, and the direct field from the emitter to the point. To improve convergence, it is noted that Equation 1 should be evaluated with an approximate visibility test, based on backface-culling [5]. Note that this visibility test should also be applied to compute $\bar{H}_{i}^{i}$ from the incident wave.

The improvements we develop in this paper all refer to Equation 1 and Equation 2, and are mostly orthogonal to each other.

\section{B. Arbitrary dielectric coatings: Solving for $J$ and $M$}

Equation 1 assumes that all materials are perfect electric conductors, through the physical optics approximation for PEC: it leads to the magnetic current depending only on the magnetic field $(\bar{J}=2 \hat{n} \times \bar{H})$, and it makes the magnetic current null $(\bar{M}=0)$. [3] alleviates this limitation at a very small extra cost by using the Leontovitch impedance boundary conditions: for highly-absorbing dielectric coatings, $\bar{M}$ can still be reasonably ignored, and $\bar{J}$ can still be computed only from $\bar{H}$.

However, arbitrary dielectric coatings are not accurately taken into account with surface impedances. Instead, both $\bar{J}$ and $\bar{M}$ have to be computed as soon as the coating does not absorb much of the incident energy. Moreover, for any layered coating of dielectrics on a metallic surface, it is possible to compute the Fresnel reflection coefficients that represent the scattering properties of the coating. Still using physical optics approximations, we use Kottler equations and the material's Fresnel reflection coefficients to reformulate IPO.

First, the electric and magnetic currents induced by an arbitrary electric and magnetic fields are:

$$
\begin{aligned}
\bar{J}(\bar{H}, \bar{r}) & =\hat{n} \times\left(\bar{H}_{i}(\bar{r})+\bar{H}_{r}(\bar{r})\right) \\
\bar{M}(\bar{E}, \bar{r}) & =-\hat{n} \times\left(\bar{E}_{i}(\bar{r})+\bar{E}_{r}(\bar{r})\right) .
\end{aligned}
$$

$\bar{E}_{r}(\bar{r})$ and $\bar{H}_{r}(\bar{r})$ are the reflected fields, calculated from the matrix expression of the Fresnel reflection coefficients of the material at $\bar{r}$.

Second, the incident electric and magnetic fields induced by current distributions $\bar{J}$ and $\bar{M}$ are:

$$
\begin{aligned}
& \bar{E}_{i}(\bar{J}, \bar{M})(\bar{r})=-j k f_{S} E\left(\bar{r}^{\prime}, \bar{r}\right) G\left(\bar{r}-\bar{r}^{\prime}\right) d S^{\prime} \\
& \bar{H}_{i}(\bar{J}, \bar{M})(\bar{r})=-j k \int_{S} \bar{H}\left(\bar{r}^{\prime}, \bar{r}\right) G\left(\bar{r}-\bar{r}^{\prime}\right) d S^{\prime} \\
& \bar{E}\left(\bar{r}^{\prime}, \bar{r}\right)=Z_{0} \alpha_{1} J\left(\bar{r}^{\prime}\right)+Z_{0} \alpha_{2}\left(\hat{R} \cdot \bar{J}\left(\bar{r}^{\prime}\right)\right) \hat{r}-\alpha_{3} \hat{R} \times \bar{M}\left(\bar{r}^{\prime}\right) \\
& \bar{H}\left(\bar{r}^{\prime}, \bar{r}\right)=\frac{\alpha_{1}}{Z_{0}} \bar{M}\left(\bar{r}^{\prime}\right)+\frac{\alpha_{2}}{Z_{0}}\left(\hat{R} \cdot \bar{M}\left(\bar{r}^{\prime}\right)\right) \hat{r}+\alpha_{3} \hat{R} \times \bar{J}\left(\bar{r}^{\prime}\right) \\
& \alpha_{1}=1-\frac{j}{k R}-\frac{1}{k^{2} R^{2}} \\
& \alpha_{2}=-1+\frac{3 j}{k R}+\frac{3}{k^{2} R^{2}} \\
& \alpha_{3}=1-\frac{j}{k R} \\
& G(\bar{R})=\frac{e^{-j k R}}{4 \pi R}
\end{aligned}
$$

with $\bar{R}=\bar{r}-\bar{r}^{\prime}$ and $R=\|\bar{R}\|$.

Putting all together, IPO with arbitrary dielectric coatings consists in finding $\bar{J}$ and $\bar{M}$ that match the following equations:

$$
\begin{aligned}
\bar{J}(\bar{r}) & =\bar{J}\left(\bar{H}^{i}, \bar{r}\right)+\bar{J}(\bar{H}(\bar{J}, \bar{M}), \bar{r}) \\
\bar{M}(\bar{r}) & =\bar{M}\left(\bar{E}^{i}, \bar{r}\right)+\bar{M}(\bar{E}(\bar{J}, \bar{M}), \bar{r})
\end{aligned}
$$

Once the two distributions are found, the field radiated toward any point is computed using Equation 6 and Equation 7. 
The two equations above have the same form as the original IPO equation: a constant term linked to the incident wave, and a second term corresponding to a propagation of the current distribution. This motivates the fact that in the remaining of this paper, we simply handle a single distribution $J \bar{M}$, which is simply an "interleaved" current distribution where each element has both an electric component and a magnetic component.

\section{Improved solver: Preconditioned GMRESR}

Both Equation 1 or the system of equations $(9,10)$ can be expressed with a propagation operator $T$, such that, for any current distribution $\bar{J}$ or $J \bar{M}$ we have

$$
\bar{D}=\bar{D}^{i}+T \bar{D} \text {. }
$$

with $\bar{D}^{i}$ is a constant distribution representing the current induced by the incident wave coming from SBR. $T$ has the form $\hat{n} \times f \ldots d S^{\prime}$, and is linear in the current distribution it operates on.

The simplest way to solve Equation 11 is to use a fixedpoint approach: from an initial distribution $\bar{D}_{0}$ (which may be full of zeros), apply Equation 11 to obtain $\bar{D}_{1}$, and so on until $\bar{D}_{n+1}$ is close enough to $\bar{D}_{n}$. As shown in [5], this approach is not optimal and is not even robust. Many cases lead to failure, depending on $\bar{D}_{0}$ and the object IPO is run on. A better approach is to consider Equation 11 as a linear system to solve. As a matter of fact, Equation 11 can be rewritten as:

$$
(I-T) \bar{D}=\bar{D}^{i}
$$

where $I$ is the identity operator. This rewriting enables the use of all methods from linear system resolution litterature. Directly inverting $I-T$ would require very long precomputations, not necessarily balanced by the number of distributions to find. Therefore, we focus on iterative solvers. [5] presents a solver for IPO which, as identified by the authors, is very similar to a particular and reduced case of a standard and well recognized method, GMRES [7]. [5] shows the tremendous potential of using these kind of methods, although the solver they develop still requires a large number of iterations for difficult cases. We push their idea further, by finding a compromise between algorithmic complexity and gain in number of iterations and robustness.

A large number of different methods exist in the litterature, and presenting in detail these methods and their extensions is by large out of the scope of this paper. However, these methods share a common process: they use a loop, which consists in three steps: first, generating a guess $\bar{D}_{n}$ (from any data it might have stored), computing the residual $(I-T) \bar{D}_{n}$, and comparing it with $\bar{D}^{i}$ to dedice whether it is close enough or not. The differences between the methods mostly come from how the guess is generated.

There is not a single method which outruns all the others, it depends on the specific problem at hand. Iterative solvers have been extensively studied for MoM implementations. As IPO is a simplified case of MoM, it shares a lot of common numerical properties, and results that apply to MoM can also be used for IPO. [8] shows that, in the context of MoM, a good combination is a GMRES-like method and a nearfield preconditioner. Preconditioning consists in changing the linear system in a way that it is easier to solve, at the cost of some extra resolutions of simpler problems. As summarized in [8], intensive studies have been made around preconditioning for MoM, and very elaborate schemes have been developed. However, IPO does not involve problems as ill-conditionned as in MoM. It is thus not worth implementing complex methods such as incomplete-LU factorisations or sparse-approximateinverse approaches. Instead, a simple approach consists in generating the next guess $\bar{D}_{n}$ by first solving a near-field problem, where only the most important contributions (the ones that are 2-3 lambdas away, represented by an operator $T^{N F}$ ) are taken into account. The generation of the guess then becomes: generate $\bar{D}_{n}^{*}$ with GMRES, solve $\left(I-T^{N F}\right) \bar{D}_{n}^{*}=$ $\bar{D}^{i}$ with another GMRES, use this solution as guess $\bar{D}_{n}$. With this process, each guess is first optimized to better take into account the most important contributions. This preconditioning method is easy to implement, as it is a restricted case of $T$. However, because of the nature of the preconditioner, it requires a special kind of GMRES-like method, called "flexible" GMRES methods. [8] uses a variant of GMRES called FGMRES [9]. In our implementation, we prefer the truncated-GMRESR solver [10], which seems to behave a little better. Practically, we use truncated-GMRESR(10) as outer solver, and standard GMRES(2) with a maximum of 3 iterations to approximately solve $I-T^{N F}$.

\section{Propagation: Accurate geometry-aware propagation}

The propagation phase, which consists in applying the operator $T$, is critical for results accuracy and global resolution efficiency.

Propagation accuracy: Equation 1 has a term in $1 / R^{2}$ which can become problematic when patches are close. The situation is even worse in the dielectric formulation we use, as terms in $1 / R^{3}$ are present. Patches do not overlap, and backface culling prevents two collocated patches pointing in opposite directions to contribute to each others, therefore the case $R=0$ does not occur. However, when computing patch-to-patch interaction $\left(\bar{E}\left(\bar{r}^{\prime}, \bar{r}\right)\right.$ and $\left.\bar{H}\left(\bar{r}^{\prime}, \bar{r}\right)\right)$, the $1 / R^{2}$ or $1 / R^{3}$ terms lead to strong variations of the integrand over the source patch (the discretized surface element around $\bar{r}^{\prime}$ ) when the destination patch (the discretized surface element around $\bar{r}$ ) is close ( $R$ close to or smaller than $2 \lambda)$. Thus, a small variation in the patch center placement leads to a large variation of the contribution when only computing center-tocenter contributions, making the result highly sensitive to the patches sampling. This justifies the use of specific methods to properly integrate the field from the source patch to the destination patch when they are close. One such method is the third-degree polynomial transformation [11], which transforms highly varying and quasi-singular integrands with $1 / R^{3}$ terms into regular ones by introducing a transform whose jacobian contains a $R^{3}$ term, cancelling the $1 / R^{3}$ term. Combined with Gauss-Legendre integration, this gives high-accuracy results even with very short $R$ distances, while using a low number of points for Gauss-Legendre integration. Moreover, [11] gives insights that allow us to use a different number of points for Gauss-Legendre integration (or even no Gauss-Legendre integration) depending on the relative configuration of the two patches, for an optimal accuracy/performance compromise.

Propagation efficiency: Applying the operator $T$ is by 
large the most time-consuming part of the process: for a discretisation in $N$ patches, $N^{2}$ patch-to-patch interactions have to be computed. For large objects $\left(20 \times 20 \times 20 \lambda^{3}\right.$ or larger), up to 30000 patches can be generated, leading to nearly 1 billion interactions to compute at each propagation. This computation can require tens of minutes on the CPU, and makes IPO impractical for RCS computations, as a large number of IPO resolutions are needed, each requiring several propagation phases.

The propagation phase can be directly parallelized on the GPU thanks to its matrix-vector multiplication nature. A direct adaptation optimized for GPU computations (minimizing memory accesses) scales well with the GPU capacity. The observed speedup between pure CPU execution and GPU execution is roughly 40 on a laptop desktop with a core i5 and NVidia GT550M GPU, 100 on a desktop PC with core i7 and a NVidia GTX480 GPU, and 200 on a core $17+$ NVidia Titan. This makes a 20-minutes-long computation take respectively 30, 12 and 6 seconds. However, this straightforward implementation is far from optimal, and can be optimized for GPU by taking into account both the hardware architecture of GPUs and the nature of the equations behind IPO. Both Equation 1 and the more complex propagation equations (6, 7) have a common property: it is zero for any point in the tangent plane of the source patch. Adding backface-culling, this means that the interaction from a patch $p_{1}$ to a patch $p_{2}$ is zero if $p_{2}$ belongs to the tangent plane of $p_{1}$, or if backface culling applies (a patch does not receive contributions coming from its backface, but it can emit in the backface). Note that the interaction "sparsity pattern" is not symmetric (and thus the sparsity pattern of $T$ is not symmetric), because backface culling is not symmetric.

GPUs perform computations per blocks of $N_{t}$ threads, most with $N_{t}=32$. To exploit this in our implementation, we cut the operator $T$ in blocks of $N_{t} \times N_{t}$ pairs of patches, each block of pairs of patches being processed by one block of $N_{t}$ threads. For each block, we precompute a "status" depending on the patch-to-patch interactions in the block: 2 if at least one interaction requires Gauss-Legendre integration, 1 if there is at least one non-zero interaction but no one requires GaussLegendre integration, and 0 if all interactions are zero. This last case happens frequently when patches are "correlated" in terms of orientations or placement in the patches array (for instance if all the patches of a plate are grouped in the patches array). A first optimization is to compute only the blocks with a non-zero status, which saves a lot of useless computations. A second optimization is to maximise the potential parallelism of GPUs, even if the Gauss-Legendre integration CUDA code consumes a large number of registers, thus decreasing the amount of effective parallelism the GPU can achieve. To overcome this, we run a specific code with Gauss-Legendre integration for all blocks with status 2, and we use a code without Gauss-Legendre integration for all the blocks with status 1 (the majority). Compared to the straightforward CUDA implementation, these two optimisations give us an extra performance factor of 8 (roughly decomposed in a factor 2 for the first optimization and a factor 4 for the second).

Therefore, with high accuracy and without adding any approximation, the global speedup factor compared to standard propagation on CPU is around 300 for the laptop (20min $\rightarrow$

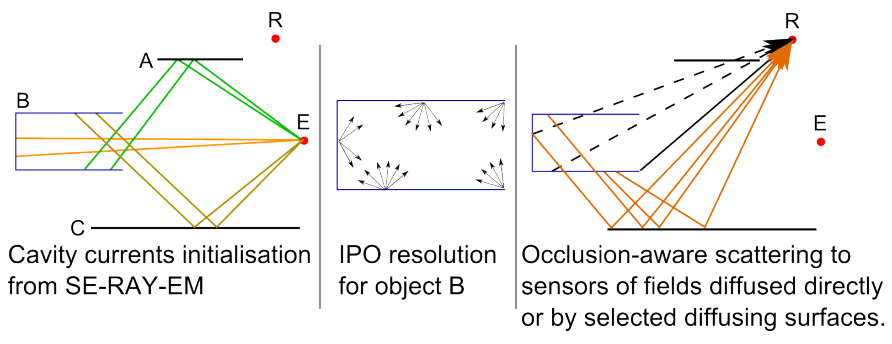

Fig. 1. Hybridization between SBR and IPO for a scene with emitter E, reception point $\mathrm{R}$, and three objects $\mathrm{A}, \mathrm{B}$ and $\mathrm{C}$. $\mathrm{B}$ is the object handled by IPO, $\mathrm{A}$ and $\mathrm{C}$ are handled only by the SBR. In the final step, A re-radiates toward the reception point, and also toward $\mathrm{C}$ which diffuses the fields toward the reception point. The dashed lines are the contributions that are occluded by an object not handled by IPO, and must therefore be ignored.

$3.5 \mathrm{~s}), 800$ for the core $\mathrm{i} 7+\mathrm{GTX} 480(20 \mathrm{~min} \rightarrow 1.5 \mathrm{~s})$, and 1600 for the core $i 7+$ Titan $(20 \mathrm{~min} \rightarrow 0.75 \mathrm{~s})$.

\section{E. Hybridization: Direct current accumulation}

Our implementation of IPO is integrated into a SBR, and coupled in a one-way process illustrated by Figure 1: first, the SBR is used to shoot rays throughout all the scene and make them bounce around, computing the field reflected by the scene toward the reception points. When a ray hits an object handled by IPO, it is stopped. Once all the rays have been traced, IPO is used to compute the current distribution of the object based on the initial current distribution obtained from the SBR phase, and these currents reradiate toward the reception points, and possibly toward other objects explicitely selected by the user. These objects diffuse the IPO fields directly toward the reception point.

IPO relies on an initial current distribution $\bar{J}^{i}$ or $J \bar{M}^{i}$, which represents the fields from the SBR that arrived on the object. This initial current distribution is classically obtained using a virtual interface surrounding the object (or at its aperture for cavities) [1]. This interface captures the SBR fields at regular points on a grid by accumulating it as current. Currents accumulated at these points then reradiate toward the cavity to yield the initial distribution, using the Kirchhoff approximation. However, this representation of the SBR fields can not capture complex incident fields, such as the ones arising in common applications of SBR, where complex occlusions and multiple bounces make the incident field highly localized and multi-directional.

In order to capture arbitrarily complex incident fields, we store all the rays that hit the IPO geometry (or any surrounding interface). As the objects handled by IPO are typically small compared to the rest of the scene, the number of incident rays is kept reasonable, making the memory cost negligible. We then compute the contribution of each ray to each patch. Even if the number of incident rays is small, making a test between all rays and all patches would be too costly. Therefore, we build a spatial subdivision structure on the incident rays, whose leaves indicate which incident rays go through the region of space the leaf covers. An important point is that the only visibility criterion used during the IPO resolution is backfaceculling. Therefore, the rays contribute to all patches that face the ray, not only the patches that directly see the ray's origin. 
As a consequence, the rays have to be considered as semiinfinite when building the spatial structure. Then, for each patch, we find the leaf in the spatial subdivision structure that contains it, and add the contributions of the rays the leaf contains to the initial current distribution, using Equation 4 and Equation 5

\section{APPLICATIONS}

IPO validation: Our dielectric IPO formulation has been validated on standard and well known cases such as dielectric dihedrons or trihedrons.

We have tested our code on various cavities for which exact results were available, with different shapes ranging from straight cylindrical cavities to S-bend cavities [12], [13]. Although IPO ignores some effects, results are in very good accordance with the results from exact methods, while SBR results strongly differ.

The test case 2 b of JINA98 [13], called "WAGGON cavity" and processed by ONERA, is a cavity that has been designed specifically to test MoM and asymptotic methods implementations. We processed it at $10 \mathrm{GHz}$. At this frequency, the cavity is $10 \lambda$ long and the aperture is $5 \lambda$ wide at its maximum. We used IPO to handle the interior of the cavity, while the outside was managed only by SBR, therefore enormously accelerating computations when the cavity was not reached. Figure 2 shows the accuracy of the IPO method for this case, compared to ONERA's Maxwell3D softwave, which implements a MoM solver. Although some differences are present, additional tests showed that they are mostly due to effects that are not accounted for by IPO, such as edges diffraction on the inner sharp corners of the cavity, or the effect of edges currents which are non-negligeable for small objects. This last effect tends to disappear for this cavity at higher frequencies.

Hybridization and performances: We used our hybridized SBR-IPO implementation to compute a $360^{\circ} \mathrm{RCS}$ of a military aircraft at a frequency of $10 \mathrm{GHz}$, with one RCS computation every degree. The interior of the tailpipe and the interior of the air intake of the aircraft, shown in Figure 3, were handled with IPO, all the rest was handled by standard SBR. The aircraft is more than $350 \lambda$ long and $200 \lambda$ large, which makes it impossible to handle completely in IPO. However, as the tailpipe and air intake are much smaller, we can still benefit from precise N-PO computations for these parts, while the other parts, which do not present cavity-like properties, are already accurately handled by standard SBR. Our hybridization scheme also allows us to seamlessly integrate the aircraft in a much wider and complex environment, with landscape, buildings, etc.

The tailpipe is $20 \times 20 \times 20 \lambda^{3}$ large, and requires 26640 patches for discretization at a resolution of 4 points per $\lambda^{2}$. One propagation phase on the tailpipe requires 0.40 s on a Titan GPU (3.12s on a laptop GT550M GPU, 15.5 minutes on CPU), and an average of 16 iterations were required to reach a 0.1 residual error. The air intake is $24 \times 15 \times 12 \lambda^{3}$ large, and requires 15242 patches for 4 points per $\lambda^{2}$. One propagation phase requires $0.12 \mathrm{~s}$ on a Titan GPU (9 seconds on a laptop GT550M GPU, 4.5 minutes on CPU), and an average of 12 iterations were required to reach a 0.1 residual error.
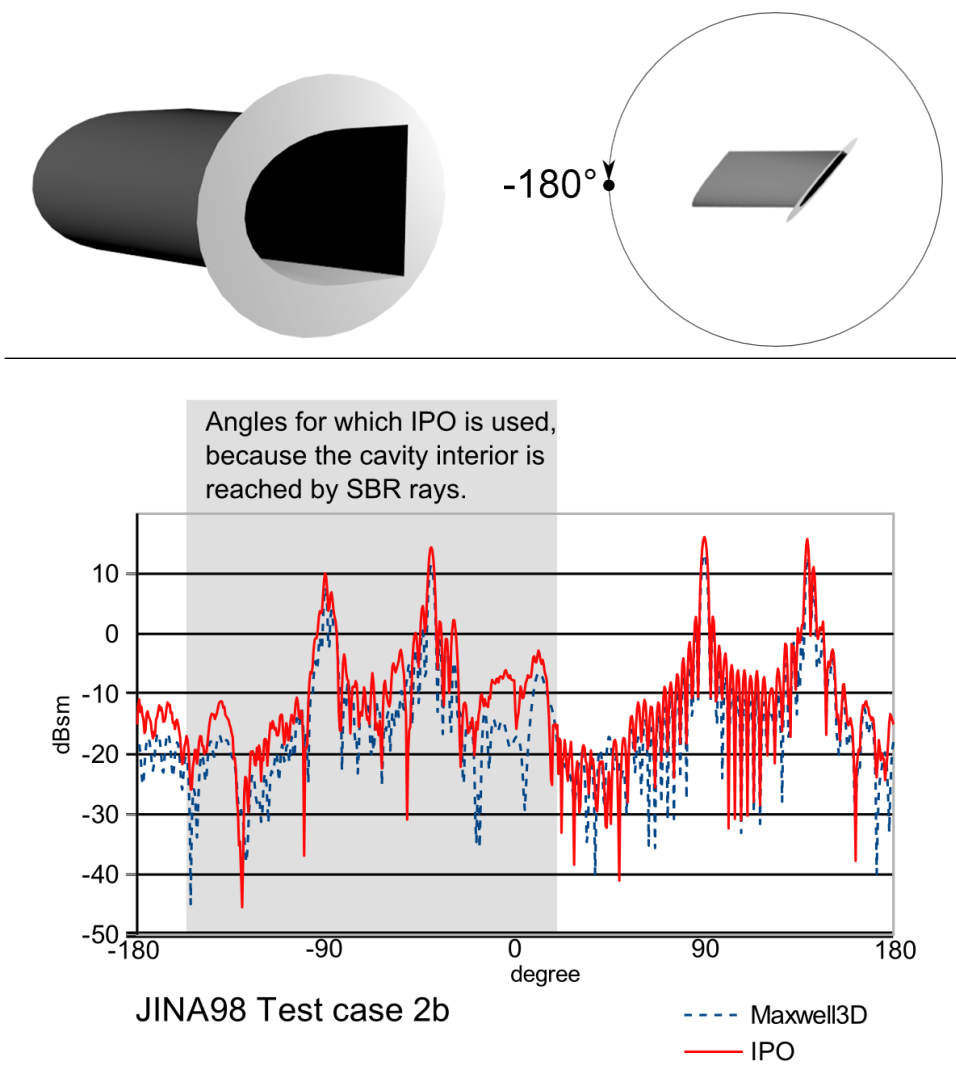

Fig. 2. JINA WAGGON cavity test case. Top left: 3D representation of the cavity, $20 \lambda$ long, $10 \lambda$ aperture. Top right: RCS sensor setup, leaving from $-180^{\circ}$ to $180^{\circ}$ by $0.5^{\circ}$ steps. Bottom: RCS results of IPO in dotted-line against exact methods in continuous line.

The total computation time for the complete RCS with IPO is 20 minutes on a Titan GPU, 2h30min on the laptop, and would have taken the non-acceptable time of a month (31 days to be more precise) on CPU. IPO was used for 235 angles, no rays having reached the interior of the cavities for the other angles. Although the computation time without IPO is a matter of seconds, the RCS comparison in Figure 3 clearly shows the impact of handling both the tailpipe and the air intake with IPO on the RCS.

\section{CONCLUSION}

The main purpose of this paper is to demonstrate the benefit of introducing the IPO approach in our RF simulation workbench that is dedicated to high frequencies and large radar scenes, with different kinds of entities and consequently different kinds of materials. This is why we emphasize on the implementation of IPO that is specifically designed for efficient computation and for a large application domain in terms of materials. Besides a massively parallel implementation has been selected in order to take advantage of the wide computing power available on GPUs. Nevertheless IPO cannot be applied on the whole scene at least for a matter of computation time. Moreover IPO is not needed everywhere: for a major part of the scene the SBR approach implemented in SE-RAY-EM is sufficient. So an accurate hybridization between SBR and IPO is presented in this paper as the relevant solution for addressing large scenes where IPO is applied locally only where it is necessary. Our hybrid approach is already available in our RF 

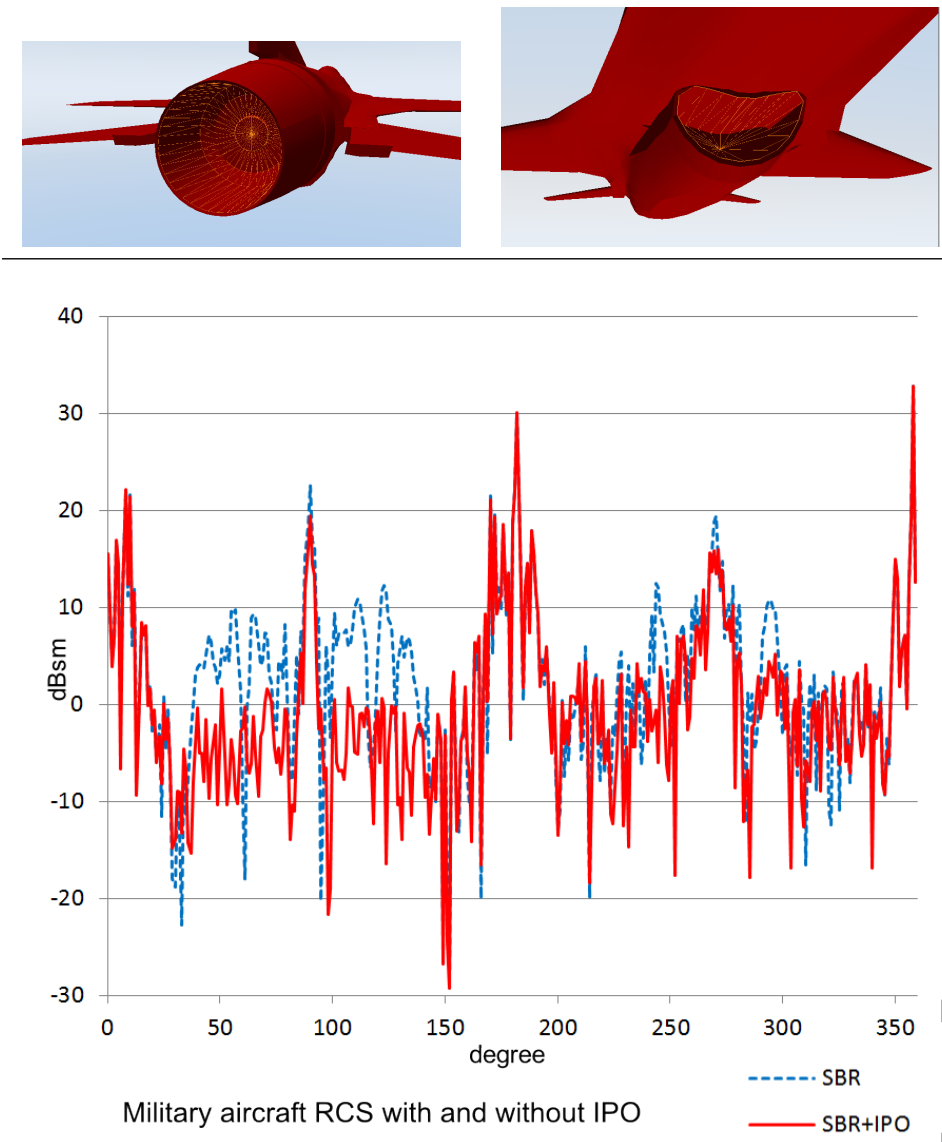

Fig. 3. Top left: Tailpipe of the aircraft. Top right: Air intake. In both cases, the region highlighted in wireframe is the zone handled by IPO. Bottom: Comparison of RCS results without IPO (dashed line) and with IPO (continuous line). The tailpipe is seen around $90^{\circ}$, the air intake is seen around $270^{\circ}$.

simulation software package, SE-Workbench-RF, but it still needs to be improved especially regarding the following issues:

- Taking into account the roughness of surfaces

- Taking efficiently into account a geometry of arbitrary shape that can be non-convex and contain geometry not handled by IPO

- Taking efficiently into account larger objects, in order to be able to address larger scenes with IPO such as the cabin of an aircraft. For that, multipole-like methods and/or asymptotic frequency formulations of currents are potential candidates that need to be further investigated.

\section{ACKNOWLEDGMENT}

This work has been funded by the AMPERE/ANR-11MONU-015 project.

\section{REFERENCES}

[1] F. Obelleiro-Basteiro, J. Rodriguez, and R. Burkholder, "An iterative physical optics approach for analyzing the electromagnetic scattering by large open-ended cavities," Antennas and Propagation, IEEE Transactions on, vol. 43, no. 4, pp. 356-361, 1995.
[2] R. Burkholder, C. Tokgoz, C. Reddy, and P. Pathak, "Iterative physical optics: its not just for cavities anymore [em wave propagation]," vol. 1A, pp. 18-21 Vol. 1A, 2005.

[3] F. Obelleiro-Basteiro, M. G. Araújo, and J. Rodriguez, "Iterative physical-optics formulation for analyzing large waveguides with lossy walls," Microwave and Optical Technology Letters, vol. 28, no. 1, pp. 21-26, 2001.

[4] R. Burkholder and T. Lundin, "Forward-backward iterative physical optics algorithm for computing the rcs of open-ended cavities," Antennas and Propagation, IEEE Transactions on, vol. 53, no. 2, pp. 793-799, 2005.

[5] R. Burkholder, "A fast and rapidly convergent iterative physical optics algorithm for computing the res of open-ended cavities," ACES Journal, vol. 16, no. 1, 2001.

[6] R. Burkholder, P. Pathak, and C. Reddy, "Asymptotic phase-front extraction applied to iterative physical optics for electrically large multibounce problems," in Antennas and Propagation Society International Symposium, 2004. IEEE, vol. 4, 2004, pp. 4447-4450 Vol.4.

[7] Y. Saad and M. Schultz, "Gmres: A generalized minimal residual algorithm for solving nonsymmetric linear systems," SIAM Journal on Scientific and Statistical Computing, vol. 7, no. 3, pp. 856-869, Jul. 1986. [Online]. Available: http://dx.doi.org/10.1137/0907058

[8] L. Grel and T. Malas, "Iterative near-field preconditioner for the multilevel fast multipole algorithm," SIAM Journal on Scientific Computing, vol. 32, no. 4, pp. 1929-1949, 2010. [Online]. Available: http://epubs.siam.org/doi/abs/10.1137/09076101X

[9] Y. Saad, "A flexible inner-outer preconditioned gmres algorithm," SIAM Journal on Scientific Computing, vol. 14, no. 2, pp. 461-469, Mar. 1993. [Online]. Available: http://dx.doi.org/10.1137/0914028

[10] H. Van der Vorst and C. Vuik, "Gmresr: a family of nested gmres methods," Numerical Linear Algebra with Applications, vol. 1, no. 4, pp. 369-386, 1994. [Online]. Available: http://dx.doi.org/10.1002/nla.1680010404

[11] J. Telles and R. Oliveira, "Third degree polynomial transformation for boundary element integrals: further improvements," Engineering analysis with boundary elements.

[12] H. Ling, R. Chou, and S. Lee, "Shooting and bouncing rays: calculating the res of an arbitrarily shaped cavity," Antennas and Propagation, IEEE Transactions on, vol. 37, no. 2, pp. 194-205, Feb 1989.

[13] "Workshop EM JINA 98, test case number 2b, Waggon cavity, Nice, France," Nov 1998. 